\title{
On Basis Property of Root Functions For a Class Second Order Differential Operator
}

Volkan Ala ${ }^{\dagger}$, Khanlar R. Mamedov

Mersin University, Arts and Letters Faculty, Department of Mathematics, 33343, Mersin

Turkey

Submission Info

Communicated by Hacı Mehmet Baskonus

Received August 1st 2019

Accepted September 30th 2019

Available online March 31st 2020

\section{Abstract}

In this work we investigate the completeness, minimality and basis properties of the eigenfunctions of one class discontinuous Sturm-Liouville equation with a spectral parameter in boundary conditions.

Keywords: Basis Property, Sturm-Liouville,Eigenvalues

AMS 2010 codes: 34L10,34B24,47E05

\section{Introduction}

We consider the boundary value problem for the differential equation on the interval $[-1,1]$

$$
\ell(u): \equiv-u^{\prime \prime}+q(x) u=\lambda w(x) u,
$$

with the boundary conditions

$$
\begin{gathered}
L_{1}(u):=u(-1)+h u^{\prime}(-1)=0, \\
L_{2}(u):=\left(\beta_{1} u(1)-\beta_{2} u^{\prime}(1)\right)+\lambda\left(\widetilde{\beta}_{1} u(1)-\widetilde{\beta}_{2} u^{\prime}(1)\right)=0,
\end{gathered}
$$

and the transmission conditions

$$
\begin{gathered}
L_{3}(y):=\gamma_{1} u(-0)-\delta_{1} u(+0)=0, \\
L_{4}(y):=\gamma_{2} u^{\prime}(-0)-\delta_{2} u^{\prime}(+0)=0,
\end{gathered}
$$

where

$$
w(x)=\left\{\begin{array}{l}
w_{1}^{2},-1 \leq x<0 \\
w_{2}^{2}, 0<x \leq 1
\end{array}\right.
$$

\section{${ }^{\dagger}$ Corresponding author.}

Email address: volkanala@mersin.edu.tr

\section{sciendo}


$w_{1} \neq w_{2}$, the real valued function $q(x) \in C([-1,0) \cup(0,1])$ and has finite limits $q( \pm 0)=\lim _{x \rightarrow \pm 0} q(x), \lambda$ is complex parameter and we assume that $h, w_{i}, \beta_{i}, \widetilde{\beta}_{i}, \gamma_{i}, \delta_{i}(i=1,2)$ are real numbers, $\gamma_{i}, \delta_{i}$ are positive coefficients, $\left|\beta_{1}\right|+$ $\left|\beta_{2}\right| \neq 0,\left|\widetilde{\beta}_{1}\right|+\left|\widetilde{\beta}_{2}\right| \neq 0$ and $\rho:=\beta_{1} \widetilde{\beta}_{2}-\widetilde{\beta}_{1} \beta_{2}$.

Spectral problems for the discontinuous Sturm-Liouville equations with eigenparameter dependent boundary conditions have been growing interest with physical applications and examined in [1-4]. For example, boundary value problems with the form (1)-(7) is encountered in vibrating string problems investigated in [19]. This happens whenever one applies the method of seperation of variables to solve the corresponding partial differential equation which contain a directional derivative in boundary conditions. On eigenvalues problems for second order equation with spectral parameter in the boundary conditions are considered in [5-13, 20-22]. Some self adjoint problems on eigenvalues for second order equation with spectral parameter in the boundary conditions are considered in [5-11]. The corresponding problems led to the eigenvalue problem for a linear operator acting on the space $L_{2} \oplus \mathbb{C}^{N}$, where $\mathbb{C}^{N}$ is $N$ - dimensional Euclidean space of complex numbers. In [4] for distinct cases, it is shown that the eigenfunctions of the spectral problem formed a defect basis in $L_{2}(0,1)$. In [14] Rayleigh-Ritz formula is developed for eigenvalues.

The goal of this work is to investigate the problem of completeness, minimality and basis property of the eigenfunctions of the boundary value problem (1)-(5). In this study, we introduce a special inner product in a special Hilbert space and construct a linear operator $A$ in it so that the problem (1)-(5) can be interpreted as the eigenvalue problem for $A$. Let us give the main definitions and theorem which will be used in our main results:

Definition 1. [23] A sequence $\left\{f_{j}\right\}_{j \geq 1}$ of vectors of a Hilbert space $H$ is called a basis of this space if every vector $f \in H$ can be expanded in a unique way in a series $f=\sum_{j=1}^{\infty} c_{j} f_{j}$, which converges in the norm of the space $H$.

Definition 2. [23] A basis $\left\{f_{j}\right\}_{j \geq 1}$ of $H$ is called a Riesz basis if it is obtained from an orthonormal basis by means of a bounded linear invertible operator.

Theorem 1. [24] Let A be selfadjoint compact operator or selfadjoint operator which has discrete spectrums. Then the eigenfunctions of the operator A form orthonormal basis in the Hilbert space $H$.

For example;

$$
\begin{gathered}
u^{\prime \prime}(x)+\lambda u(x)=0, \quad x \in(0,1) \\
u(0)=0, u^{\prime}(1)=d \lambda u(1), \quad d>0,
\end{gathered}
$$

this boundary value problem has only the eigenfunctions $u_{n}(x)=\sqrt{2} \sin \sqrt{\lambda_{n}} x, n=0,1,2 .$. , with positive eigenvalues found from the equation $\cot \sqrt{\lambda}=d \sqrt{\lambda}$. By deleting an arbitrary eigenfunction, one obtains a basis in the space $L_{p}(0,1) p>1$, even a Riesz bases in the case $p=2$. [4]

\section{The Operator Formulation of the Problem and Main Results}

It is convenient to represent the spectral problem (1)-(5) as an eigenvalue problem for a linear problem in a Hilbert space. We denote by $H=L_{2}[-1,1] \oplus \mathbb{C}$ the special Hilbert space of all elements

$$
\widetilde{u}=\left(\begin{array}{c}
u(x) \\
u_{1}
\end{array}\right), \widetilde{v}=\left(\begin{array}{c}
v(x) \\
v_{1}
\end{array}\right) \in H,
$$

with the inner product

$$
(\widetilde{u}, \widetilde{v})=w_{1}^{2} \gamma_{1} \gamma_{2} \int_{-1}^{0} u(x) \overline{v(x)} d x+w_{2}^{2} \delta_{1} \delta_{2} \int_{0}^{1} u(x) \overline{v(x)} d x+\frac{\delta_{1} \delta_{2}}{\rho} u_{1} \overline{v_{1}} .
$$


In the space we define the operator

$$
A \widetilde{u}=\left(\begin{array}{c}
\frac{1}{w(x)}\left[-u^{\prime \prime}+q(x) u\right] \\
\beta_{1} u(1)-\beta_{2} u^{\prime}(1)
\end{array}\right)
$$

on the domain

$$
D(A)=\left\{\begin{aligned}
\widetilde{u} \widetilde{u}=\left(u(x), u_{1}\right) & \in H, u(x), u^{\prime}(x) \in A C([-1,0) \cup(0,1]), \\
u^{\prime}( \pm 0) & =\lim _{x \rightarrow \pm 0} u^{\prime}(x), \ell(u) \in L_{2}[-1,1], \\
L_{1} u=L_{3} u & \left.=L_{4} u=0, u_{1}=\widetilde{\beta}_{1} u(1)-\widetilde{\beta}_{2} u^{\prime}(1)\right),
\end{aligned}\right\}
$$

where $A C([-1,1])$ is the space of all absolutely continuous functions on the interval $[-1,1]$. Obviously, the operator $A$ is well defined in $H$. It is clear that the spectral problem (1)-(5) is equivalent to operator equation

$$
A \widetilde{u}=\lambda \widetilde{u},
$$

and the eigenvalues of $A$ coincide with the eigenvalues of the problem (1)-(5) (see Lemma 1.4. in [5]). Also, there exists a correspondence between eigenfunctions

$$
\widetilde{u}_{k}(x) \leftrightarrow\left(\begin{array}{c}
u_{k}(x) \\
\left.\widetilde{\beta}_{1} u(1)-\widetilde{\beta}_{2} u^{\prime}(1)\right)
\end{array}\right)
$$

Lemma 2. The domain $D(A)$ of the operator $A$ is dense in the space $H$.

Proof. The proof is similar using the same method in [15]. Suppose that $\widetilde{f} \in H$ is orthogonal to all $\widetilde{g} \in D(A)$ with respect to the inner product (6), where $\widetilde{f}=\left(f(x), f_{1}\right), \widetilde{g}=\left(g(x), g_{1}\right)$. Let $\widetilde{C}_{0}^{\infty}$ denote the set of functions

$$
\Phi(x)=\left\{\begin{array}{c}
\varphi_{1}(x), x \in[-1,0) \\
\varphi_{2}(x), x \in(0,1]
\end{array}\right.
$$

where $\varphi_{1}(x) \in C_{0}^{\infty}[-1,0)$ and $\varphi_{2}(x) \in C_{0}^{\infty}(0,1]$. Since $\widetilde{C}_{0}^{\infty} \oplus 0 \subset D(A)(0 \in \mathbb{C})$, any $\widetilde{u}=(u(x), 0) \in \widetilde{C}_{0}^{\infty} \oplus 0$ is orthogonal to $\widetilde{f}$, namely,

$$
(\widetilde{f}, \widetilde{u})=w_{1}^{2} \gamma_{1} \gamma_{2} \int_{-1}^{0} f(x) \overline{u(x)} d x+w_{2}^{2} \delta_{1} \delta_{2} \int_{0}^{1} f(x) \overline{u(x)} d x=(f, u)_{1}
$$

where $(,)_{1}$ denotes inner product in $L_{2}[-1,1]$. This implies that $f(x)$ is orthogonal to $\widetilde{C}_{0}^{\infty}$ and $(f, u)_{1}=0$. Hence,

$$
(\widetilde{f}, \widetilde{g})=\frac{\delta_{1} \delta_{2}}{\rho} f_{1} \overline{g_{1}}=0 .
$$

Thus $f_{1}=0$ since $g_{1}=\widetilde{\beta}_{1} g(1)-\widetilde{\beta}_{2} g^{\prime}(1)$ can be chosen arbitrary. So $\widetilde{f}=(0,0)$. Therefore, $D(A)$ is dense in $H$.

Lemma 3. The operator $A$ is selfadjoint.

Proof. In this case integrating by parts, we obtain that $(A \widetilde{f}, \widetilde{g})$ is real. Taking into account Lemma 2, we find that the operator $A$ is symmetric in the space $H$. Boundary value problem (1)-(5) is solvable for every non-eigenvalue $\lambda$ and has discrete spectrum. Therefore the operator $A$ is symmetric and has discrete spectrum. Hence, the operator $A$ is selfadjoint in $H$.

Theorem 4. The eigenfunctions of the operator $A$ form an orthonormal basis in the space $H=L_{2}[-1,1] \oplus \mathbb{C}$. 
Proof. The operator $A$ has countable many eigenvalues $\left\{\lambda_{n}\right\}_{n=1}^{\infty}$ which have the asymptotic form [16]:

$$
\lambda_{n}=\frac{1}{w_{1}+w_{2}} \pi(n-1)+O\left(\frac{1}{n}\right), n \rightarrow \infty .
$$

Then for any number $\lambda$ which is not an eigenvalue and arbitary $\widetilde{f} \in H$ it can be found an element $\widetilde{u} \in D(A)$ satisfying the condition $(A-\lambda I) \widetilde{u}=\widetilde{f}$. Thus the operator $(A-\lambda I)$ is invertible except for the isolated eigenvalues. Without loss of generality we assume that the point $\lambda=0$ is not an eigenvalue. Then we obtain that the bounded inverse operator $A^{-1}$ is defined in $H$. Thus, the selfadjoint operator $A^{-1}$ has at most countable many eigenvalues and each one of them converges to zero at the infinity. So, the selfadjoint operator $A^{-1}$ is compact. Applying the Hilbert-Schmidt theorem to this operator we obtain that the eigenfunctions of the operator $A$ form an orthonormal basis in $H$. Theorem is proved.

Now we consider the case $\rho<0$. We assume that the operator $A$ is defined by formula (7) on the domain $D(A)$. In the space $H=L_{2} \oplus \mathbb{C}$ for $\widetilde{u}, \widetilde{v} \in H$ the scalar product is defined by formula

$$
(\widetilde{u}, \widetilde{v})=w_{1}^{2} \gamma_{1} \gamma_{2} \int_{-1}^{0} u(x) \overline{v(x)} d x+w_{2}^{2} \delta_{1} \delta_{2} \int_{0}^{1} u(x) \overline{v(x)} d x-\frac{\delta_{1} \delta_{2}}{\rho} u_{1} \overline{v_{1}} .
$$

In this case the operator $A$ is not selfadjoint in the space $H$. Therefore we introduce the operator $J$ is defined by

$$
J=\left(\begin{array}{cc}
I & 0 \\
0 & -I
\end{array}\right)
$$

where $I$ is the identity operator in $H$. Operator $J$ is selfadjoint and invertible.

In this case, the boundary value problem (1)-(5) is equivalent to eigenvalue problem for the operator pencil

$$
(B-\lambda J) \widetilde{u}=0,
$$

in the space $H$ such that $B=J A$. We obtain that (8) is equivalent to (10).

Lemma 5. The operator A is J-selfadjoint in the Hilbert space $H$.

Proof. Analogously to Lemma 2, we can show that the domain $D(A)$ is dense in space $H$. From (9) and (10) applying two times integration by parts, $(B \widetilde{u}, \widetilde{u})$ is real. Hence, the operator $B$ is symmetric. Therefore, the operator $A$ is $J$ - symmetric in the space $H$. In this case it can be proved that the operator $A$ has a discrete spectrum. Taking into consideration that the operator $B$ is symmetric we have that the operator $J A$ is selfadjoint.

Corollary 6. From the system $\left\{u_{n}\right\}_{0}^{\infty}$ one can eliminate one element so that the remaining elements will form a complete and minimal system in the space $L_{2}[-1,1]$.

Proof. By Theorem 4, the system of eigenfunctions

$$
\widetilde{u}_{n}(x)=\left\{\begin{array}{c}
u_{n}(x) \\
u_{1}
\end{array}\right\}
$$

$\left(u_{1} \in \mathbb{C}\right)$ of the operator $A$ forms an orthonormal basis in $H$. Hence, the system of the eigenfunctions $\left\{\widetilde{u}_{n}(x)\right\}_{1}^{\infty}$ is complete and minimal in the space $H$. Thus, of course, $\operatorname{codim} P=1$, then by Lemma 2.1 in [5], the system $\left\{P \widetilde{u}_{n}(x)\right\}=\left\{u_{n}(x)\right\}$ whose one element is omitted from forms a complete and minimal system in $P(H)=$ $L_{2}[-1,1]$. Hence, the eigenfunctions $\left\{u_{n}(x)\right\}_{0}^{\infty}\left(n \neq n_{0}, n_{0}\right.$ is an arbitrary nonnegative integer $)$ of the boundary problem (1)-(5) are complete and minimal system in $L_{2}[-1,1]$.

Theorem 7. The eigenfunctions of the operator A form a Riesz basis in the Hilbert space $H$. 
Proof. From the operator $J$ is a bounded operator, using the idea in Theorem 4, it can be shown that the operator $B=J A$ is invertible since the selfadjoint operator $B^{-1}$ is compact. Since the selfadjoint operator $B^{-1}$ has at most countable many eigenvalues which converge to zero at infinity. Hence the operator $B^{-1}$ is compact. Then applying Azizov-Iokhvidov theorem in section IV of [18] to operator $B$ we obtain that the eigenfunctions of the $J$ - selfadjoint operator $A$ form a Riesz basis in the space $H=L_{2} \oplus \mathbb{C}$.

Let us consider another boundary value problem for the Sturm-Liouville equation

$$
\ell u:=-p(x) u^{\prime \prime}+q(x) u=\lambda u,
$$

on $[-1,0) \cup(0,1]$ with boundary condition,

$$
\begin{gathered}
L_{1}(u):=\alpha_{1} u(-1)+\alpha_{2} u^{\prime}(-1)=0 \\
L_{2}(\lambda) u:=\lambda\left(\widetilde{\beta}_{1} u(1)-\widetilde{\beta}_{2} u^{\prime}(1)\right)+\left(\beta_{1} u(1)-\beta_{2} u^{\prime}(1)\right)=0,
\end{gathered}
$$

with transmission conditions at the point of discontinuity $x=0$,

$$
\begin{gathered}
L_{3}(u):=\gamma_{1} u(-0)-\delta_{1} u(+0)=0, \\
L_{4}(u):=\gamma_{2} u^{\prime}(-0)-\delta_{2} u^{\prime}(+0)=0,
\end{gathered}
$$

here

$$
p(x)=\left\{\begin{array}{l}
p_{1}^{2},-1 \leq x<0 \\
p_{2}^{2}, \quad 0<x \leq 1
\end{array}\right.
$$

$p_{1} \neq p_{2}$, the real valued function $q(x)$ is continuous in $[-1,0) \cup(0,1]$ and has finite limits $q( \pm 0)=\lim _{x \rightarrow \pm 0} q(x), \lambda$ is complex parameter, we assume that $h, p_{i}, \beta_{i}, \widetilde{\beta}_{i}, \gamma_{i}, \delta_{i}(i=1,2)$ are real numbers, $\gamma_{i}, \delta_{i}$ are positive coefficients, $\left|\beta_{1}\right|+\left|\beta_{2}\right| \neq 0,\left|\widetilde{\beta}_{1}\right|+\left|\widetilde{\beta}_{2}\right| \neq 0$ and $\rho:=\beta_{1} \widetilde{\beta}_{2}-\widetilde{\beta}_{1} \beta_{2}>0$.

It is convenient to represent the spectral problem (11)-(15) as an eigenvalue problem for a linear problem in a special Hilbert space. We denote by $H=L_{2}(-1,1) \oplus \mathbb{C}$ the special Hilbert space of all elements

$$
f=\left(\begin{array}{c}
f(x) \\
f_{1}
\end{array}\right), g=\left(\begin{array}{c}
g(x) \\
g_{1}
\end{array}\right) \in H,
$$

with the inner product

$$
(f, g)=\frac{1}{p_{1}^{2}} \gamma_{1} \gamma_{2} \int_{-1}^{0} f(x) \overline{g(x)} d x+\frac{1}{p_{2}^{2}} \delta_{1} \delta_{2} \int_{0}^{1} f(x) \overline{g(x)} d x+\frac{\delta_{1} \delta_{2}}{\rho} f_{1} \overline{g_{1}}
$$

In the space we define the operator

on the domain

$$
L u=\left(\begin{array}{c}
-p(x) u^{\prime \prime}+q(x) u \\
\beta_{1} u(1)-\beta_{2} u^{\prime}(1)
\end{array}\right)
$$

$$
D(L)=\left\{\begin{aligned}
\widetilde{u} \widetilde{u}=\left(u(x), u_{1}\right) & \in H, u(x), u^{\prime}(x) \in A C([-1,0) \cup(0,1]), \\
u^{\prime}( \pm 0) & =\lim _{x \rightarrow \pm 0} u^{\prime}(x), \ell(u) \in L_{2}[-1,1], \\
L_{1} u=L_{3} u & \left.=L_{4} u=0, u_{1}=\widetilde{\beta}_{1} u(1)-\widetilde{\beta}_{2} u^{\prime}(1)\right),
\end{aligned}\right\}
$$

$u( \pm 0)$ has finite limits.Now we can rewrite the problem (11)-(15) in the operator form as

$$
L u=\lambda u .
$$

The eigenvalues of the operator $L$ coincide with the eigenvalues of the spectral problem (11)-(15). 
Theorem 8. The eigenfunctions of the operator $L$ form an orthonormal basis in the space $H=L_{2}[-1,1] \oplus \mathbb{C}$.

Proof. The operator $L$ has countable many eigenvalues $\left\{\lambda_{n}\right\}_{n=1}^{\infty}$ which have the asymptotic form [17]:

$$
\lambda_{n}=\frac{\alpha_{1} \alpha_{2}}{\alpha_{1}+\alpha_{2}} \pi(n-1)+O\left(\frac{1}{n}\right), n \rightarrow \infty .
$$

Then using the idea of the proof of Theorem 4, it can be proved analogusly.

Now for the boundary value problem (11)-(15) we consider the case $\rho<0$. In the space $H=L_{2} \oplus \mathbb{C}$ for $f, g \in H$ the scalar product is defined by formula

$$
(f, g)=\frac{1}{p_{1}^{2}} \gamma_{1} \gamma_{2} \int_{-1}^{0} f(x) \overline{g(x)} d x+\frac{1}{p_{2}^{2}} \delta_{1} \delta_{2} \int_{0}^{1} f(x) \overline{g(x)} d x-\frac{\delta_{1} \delta_{2}}{\rho} f_{1} \overline{g_{1}}
$$

Since $\rho<0$, the operator $A$ is not selfadjoint in the space $H$. Therefore we introduce the operator $J$ is defined by

$$
J=\left(\begin{array}{cc}
I & 0 \\
0 & -I
\end{array}\right)
$$

where $I$ is the identity operator in $H$. Operator $J$ is selfadjoint and invertible in $H$.

In this case, the boundary value problem (11)-(15) is equivalent to eigenvalue problem for the operator pencil

$$
(B-\lambda J) \widetilde{u}=0,
$$

where $B=J L$ is symmetric and $L$ is $J-$ symmetric in the space $H$.. Similary to Lemma 5, The operator $L$ is $J-$ selfadjoint in the Hilbert space $H$ and we obtain the following results:

Theorem 9. The eigenfunctions of the operator L form a Riesz basis in the Hilbert space H.

Corollary 10. From the system $\left\{u_{n}\right\}_{0}^{\infty}$ one can eliminate one element so that the remaining elements will form a complete and minimal system in the space $L_{2}[-1,1]$.

\section{References}

[1] Tikhonov A. N., Samarskii A. A., Equations of Mathematical Physics, Pergamon, Oxford, New York, 1963.

[2] Fulton C.T., Two-Point Boundary Value Problems with Eigenvalue Parameter Contained in the Boundary Conditions, Proc. Roy. Soc. Edin., vol.77 A, pp. 293-308, 1977.

[3] Kobayashi M., Eigenvalues of Discontinuous Sturm-Liouville Problem with Symmetric Potentials, Comp. Math. Appl. vol.18, pp. 357-364, 1989.

[4] Kapustin N.Y., Moisseev E.I., A Remark on the Convergence Problem for Spectral Expansions Corresponding to a Classiscal Problem with Spectral Parameter in the Boundary Condition, Differential Equations, vol.37, no.12, pp. 1677-1683, 2001.

[5] Shkalikov A.A., Boundary Value Problems for Ordinary Differential Equations with a Spectral Parameter in the Boundary Conditions, Trudy Sem. Im. I.G. Petrovsogo, vol.9, pp.190-229, 1983.

[6] Walter J., Regular Eigenvalue Problems with Eigenvalue Parameter in the Boundary Conditions, Math. Z., vol. 133, pp. 301-312, 1973.

[7] Schneider A., Note on Eigenvalue Problems with Eigenvalue Parameter in the Boundary Conditions, Math. Z., vol. 136, pp. 163-167, 1974.

[8] Hinton D. B., An Expansion Theorem for an Eigenvalue Problem with Eigenvalue Parameter in the Boundary Condition, Quart. J. Math. Oxford, vol.30, pp. 33-42, 1979.

[9] Russakovskij E. M., Operator Treatment of Boundary Problems with Spectral Parameters Entering via Polynomials in the Boundary Conditions, Funct. Anal. Appl., vol.9, pp. 358-359, 1975.

[10] Binding P.A., Browne P.J., Siddighi K., Sturm-Liouville Problems with Eigenparameter Dependent Conditions, Proc. Edinburgh Math. Soc. vol.37, no.1, pp. 57-72, 1999. 
[11] Mamedov Kh. R., On One Boundary Value Problem with Parameter in the Boundary Conditions, Spectral Theory of Operators and Its Applications, vol.11 pp. 117-121, 1997. (In Russian)

[12] Mamedov Kh. R., On a Basic Problem for A Second Order Differental Equation with a Discontinuous Coefficient and a Spectral Parameter in the Boundary Conditions, Geometry Integrability and Quantization, pp. 218-225, 2005.

[13] Gulmamedov V. Y., Mamedov Kh. R., On Basis Property for a Boundary-Value Problem with a Spectral Parameter in the Boundary Condition, Cankaya University, Journal of Arts and Sciences, no.5, May, 2006.

[14] Belinskiy B.P., Dauer J.P., On a Regular Sturm-Liouville Problem on a Finite Interval with the Eigenvalue Parameter Appearing Linearly in the Boundary Condition, Spectral Theory and Computational Methods of Sturm-Liouville Problem, Eds. D. Hinton and P.W. Schaefer, 1997.

[15] Aiping W., Jiong S., Xiaoling H., Siqin Y., Completeness of Eigenfunctions of Sturm-Liouville Problems with Transmission Conditions, Methods and Applications of Analysis, vol. 16, no. 3, pp 299-312, 2009.

[16] Mukhtarov O., Kadakal M., Some Spectral Properties of One Sturm-Liouville Type Problem with Discontinuous Weight, Siberian Mathematical Journal, vol.46, no.4, pp. 681-694, 2005.

[17] Kadakal M., Mukhtarov O. Sh., Discontinuous Sturm-Liouville Problems Containing Eigenparameter in the Boundary Conditions, Acta Mathematica Sinica, English Series, Sep., vol. 22, no. 5, pp.1519-1528, 2006.

[18] Azizov T., Iokhidov I., Foundations of the Theory of Linear Operators in Spaces with Indefinite Metric, Moscow, Nauka, 1986. (in Russian)

[19] Anderseen R.S., The Effect of Discontinuous in Density and Shear Velocity on the Asymptotic Overtone Structure of Torsional Eigenfrequences of the Earth, Geophysical Journal Royal Astronomical Society, vol. 50, pp. 306-309, 1997.

[20] Bas E., Ozarslan R., Baleanu D., Sturm-Liouville Difference Equations Having Bessel and Hydrogen Atom Potential Type, Open Phsyics, vol. 16, no.1, 2018.

[21] Allahverdiev B.P., Tuna H., Eigenfunction Expansion for Singular Sturm-Liouville Problems with Transmission Conditions, Electronic Journal of Differential Equations, vol.3, p.p.1-10, 2019.

[22] Bairamov E., Aygar Y., Oznur G.B., Scattering Properties of Eigenparameter-Dependent Impulsive Sturm Liouville Equations, Bulletin of the Malaysian Mathematical Sciences Society, vol. 42, no.6, 3247-3259, 2019.

[23] Freiling G., Yurko V., Inverse Sturm-Liouville Problems and Their Applications, Gordon and Breach, Amsterdam, 2000.

[24] Bari N.K., Biorthogonal Systems and Bases in Hilbert Spaces, Uchen. Zap. Moskov. Gos. Univ., 178, 4: 68-107, 1951. 
